\title{
LIST OF CORRIGENDA
}

VOLUME 6

Page 117, next to last line, for " $p=\sqrt{u v "}$ read " $p=2 \sqrt{u v . " ~}$

Page 121, last column of table 2, line 9, for " $h^{2}=h^{2}$ " read " $h^{2}=h_{0}{ }^{2}$."

Page 121, last line add, "and use $h^{2}=\frac{4 h_{0}{ }^{2}\left(1-p^{2}\right)}{h_{0}{ }^{2}\left(1-4 p^{2}\right)+3}$

Page 125, line 4 , for " $=b^{2} m$ " read " $f=b^{2} m$."

Page 127, table 1, heading, for " $a^{2}=1 / 2(1+f)$ " read " $a^{2}=\frac{1}{2(1+f)}$ "

Page 130, line 7 from bottom, for " $a$ " read "as."

Page 137; line 2, for " 139 " read $\frac{\text { " } 139 "}{512}$

Page 153, next to last line, for " $p A a$ " read " $p$ ' $A a$ " and for " $1 / 2(1-p) a a$ " read " $1 / 2\left(1-p^{\prime}\right) a a . "$

Page 217, table 5, headings, for " $S_{u} T_{u}, S_{u} t_{u}, s_{u} T_{u}, s_{u} t_{u}$ " read " $C T_{u}, C t_{u}$, $c T_{u}, c t_{u} . "$

Page 448, footnote 2, line 1, for "amblyomyopic" read "amblyopic."

Page 448, footnote 2, line 2, for "fraction" read "refraction."

Page 491, table 1, column 4, line 5, for " 29 " read " 3 ."

Page 491, table 1, column 6, line 5, for " 23.39" read " 2.42."

Page 514, line 14, for " $r=.25 \sqrt{a+b+d+15 c "}$ read

$$
\text { " } r=.25 \sqrt{a+b+d-15 c . "}
$$

Page 525, line 8, for volume " 41 " read volume " $46 . "$

Page 525, line 11, for volume " 46 " read volume " 51 ."

Page 568, diagram of experiment 11, line 2, extreme right-hand, for "(white)" read "(white ?)."

Page 569, diagram of experiment 21, line 4, for " $2 B^{\prime} B^{\prime} r_{x} r_{y}$ " read " $2 B^{\prime} b^{\prime} r_{x} r_{y}$." 